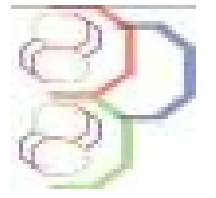

Journal of Applied Biosciences 66:5060 - 5069

ISSN 1997-5902

\title{
Effet du stress salin sur le comportement physiologique et métabolique de trois variétés de piment (Capsicum annuum l.)
}

\author{
R'him Thouraya., Tlili Imen., Hnan Imen, Ilahy Riadh , Benali Ahlem et Jebari Hager
}

Adresse professionnelle : Laboratoire d'Horticulture. Institut National de la Recherche Agronomique de Tunisie (INRAT) . Rue Hédi karray.2049.Ariana.Tinisie.

Laboratory of Horticulture. National Agricultural Research Institute of Tunisia (INRAT), Hédi karray Street .2049.Ariana. Tunisia. Tél-fax : 21671230 077.E-mail: thouraya.rhim@yahoo.fr ; bn.riadh@gmail.com ; imen tlili2008@yahoo.fr; jebari.hager@iresa.agrinet.tn

Corresponding author: R'HIM Thouraya, thouraya.rhim@yahoo.fr

Original submitted in on 10 th May 2013 Published online at www.m.elewa.org on 30th June 2013.

https://dx.doi.org/10.4314/jab.v66i0.95004

\section{RÉSUME}

Objectif : Cette étude a pour objectif de comparer le comportement aussi bien physiologique que métabolique de trois variétés de piment qui diffèrent par leur goût (doux, piquant et très piquant) et par leur origine et qui sont soumises à des conditions de stress salin.

Méthodologie et résultats : Quatre traitements salins ont été appliqués $(0,25,50,100 \mathrm{mmol}$ de $\mathrm{NaCl})$. Les résultats obtenus montrent que la salinité a un effet dépressif sur les trois variétés par une réduction de la matière sèche de la plante, de la conductance stomatique et de la teneur en chlorophylle $a, b$ et totale. Toutefois, cette réduction est plus marquée chez la variété douce de Nabeul où l'effet du sel commence à se sentir à partir de $25 \mathrm{mmol}$ de $\mathrm{NaCl}$. Pour les teneurs ioniques $\left(\mathrm{K}^{+}, \mathrm{Ca}^{2+}\right.$ et $\left.\mathrm{Na}^{+}\right)$.l'analyse statistique n'a pas montré de différence significative pour les teneurs en $\mathrm{Ca}^{2+}$ et en $\mathrm{K}^{+}$chez la variété très piquante Midass quelque soit le traitement salin appliqué, alors que le taux potassique et calcique des feuilles a diminué sous l'effet de la salure essentiellement pour la variété douce Nabeul. En outre, le stress salin a entraîné une augmentation significative de la teneur en $\mathrm{Na}^{+}$des trois variétés de piment. La teneur la plus faible en $\mathrm{Na}^{+}$est enregistrée chez « Midass », qui n'a présenté un effet significatif qu'à partir du traitement T4 (100 mmol). La variété douce Nabeul a montré une sensibilité plus grande vis-à-vis du sel puisque l'ion $\mathrm{Na}+$ s'accumule rapidement dans les feuilles à partir de $25 \mathrm{mmol}$ de $\mathrm{NaCl}$. Le rapport de sélectivité $\mathrm{K}^{+} \mathrm{Na}^{+}$a été plus discriminant entre les variétés : Midass a présenté le rapport de sélectivité le plus élevé et Nabeul le rapport de sélectivité le plus faible

Conclusion et application : La variété Nabeul qui est de type piment doux est plus sensible au sel que les variétés Beldi et Midass qui sont piquantes. Ceci confirme l'importance des choix des génotypes pour la tolérance à la salinité.

Mots clé : salinité, piment, conductance stomatique, métabolites 
Abstract:

Salt stress effect on physiological and metabolical behavior of three pepper varieties (capsicum annuum .)

Objective: This study aims to compare the physiological and metabolic behaviour of three pepper varieties namely 'Nabeul', 'Beldi' and 'Midass' that differ in taste (sweet, spicy and very spicy) which were subject to salt stress conditions.

Methodology and results: Four level of salt $(0,25,50,100 \mathrm{mmol}$ de $\mathrm{NaCl})$ was compared. Results showed that salinity exerted a depressive effect on the three pepper varieties. This depressive effect was characterized by a reduction of the dry matter, on the stomatal conductance and on the chlorophyll content ( $a, b$ and total). However, this reduction was more pronounced in 'Nabeul' where the effect of salt starts to be obvious at 25 $\mathrm{mmol}$ of $\mathrm{NaCl}$. Regarding the ionic content $\left(\mathrm{K}^{+}, \mathrm{Ca}^{2+}\right.$ and $\left.\mathrm{Na}^{+}\right)$, no significant difference was obtained for the content of $\mathrm{Ca}^{2+}$ and in $\mathrm{K}^{+}$in 'Midass' under all the studied saline treatment. However, in 'Nabeul' leaves, the content of $\mathrm{Ca}^{2+}$ and in $\mathrm{K}^{+}$decreased under the effect of different salinity treatments. In addition, the saline stress also caused a significant increase in the content of $\mathrm{Na}^{+}$in all the studied pepper varieties. The lowest $\mathrm{Na}^{+}$value was recorded for 'Midass', where a significant effect was only obtained under T4 treatment (100 $\mathrm{mmol}$ ). However, in 'Nabeul', $\mathrm{Na}^{+}$accumulated quickly in the shoots under lower salinity levels starting from the selectivity ratio $\left(\mathrm{K}^{+} / \mathrm{Na}^{+}\right)$decreased with increasing saline stress. This ratio was more selective between the varieties. Therefore, even with the accumulation of $\mathrm{Na}^{+}$, 'Midass' presented the highest selectivity ratio and Nabeul the lowest one.

Conclusion and application: Beldi and Midass hot variety peppers are more tolerant to salinity stress than sweet peppers. These data confirm the important role played by genetic background in salt tolerance.

Keywords: salinity, pepper, stomatal conductance, metabolite

\section{INTRODUCTION}

Dans les régions arides et semi arides, la salinité constitue une contrainte majeure à la productivité et au développement agricole (Rozema and Flowers, 2008 ; Abdel Latef, 2010). Actuellement, sur 1.5 milliard d'hectares de terre cultivée dans le monde, environ 77 millions d'hectares (5\%) sont affectés par la teneur excessive en sel (Sheng et al., 2008). Ce chiffre ne cesse d'augmenter d'une année à l'autre à la mauvaise qualité de l'eau d'irrigation (Pasternak and Malach, 1994, Villa-Castorena et al., 2003), à l'intensification des cultures ( Ghassemi et al.,1995) et à l'utilisation démesurée des fertilisants chimiques chez plusieurs espèces cultivées particulièrement chez celles cultivées sous serre (Shannon and Grieve, 1999). Le piment (Capsicum. Annuum L.) est considéré parmi les espèces légumières sensibles (Cornillon et Palloix, 1997 ; Hakan et al., 2006) ou modérément sensibles ( Navarro et al., 2010) à la salinité. Des expériences faites sur la variété Somontano de piment a montré qu'une concentration de $30 \mathrm{mM}$ de $\mathrm{NaCl}$ diminue la production de la matière sèche de $23 \%$ par rapport aux plantes non traitées (Rubio et al, 2009) De nombreux auteurs (Chartzoulakis et Klapaki, 2000 ; Zribi, 2009) ont remarqué que doses de $\mathrm{NaCl}$ supérieures à $150 \mathrm{mM}$ réduisent fortement la croissance végétative et causent des symptômes de brulures et de toxicité. Chartzoulakis et Klapaki, (2000); Navarro et al., (2003), Cabanero et al, (2004) ; Munns et Tester (2008) ; Niu et al.,(2010) ont rapporté que la réduction de la croissance de la plante est due aux diminutions du potentiel osmotique dans le sol, de la conductance stomatique; de la photosynthèse et aussi à l'augmentation de la concentration des ions $\mathrm{Na}^{+}$et $\mathrm{Cl}^{-}$qui atteignent des niveaux toxiques pour la plante. En effet, la salinité est susceptible de perturber la nutrition minérale des plantes en interférant avec le prélèvement de certains éléments essentiels comme le potassium et le calcium et ceci soit par substitution, soit par compétition au niveau des sites d'absorption membranaire (Zid et Grignon, 
1991). De plus, l'augmentation de $\mathrm{NaCl}$ diminue l'absorption du potassium et du calcium et interfère avec leurs fonctions physiologiques (Zhu, 2000; Yoshida, 2002). Par conséquent, la capacité des génotypes à maintenir des niveaux plus élevés de $\mathrm{K}^{+}$ et de $\mathrm{Ca}^{2+}$ et de faibles niveaux de $\mathrm{Na}^{+}$dans les tissus est l'un des mécanismes clés contribuant à l'expression de la tolérance au sel. En effet, Mansour (2003) et Zeng (2003) ont signalé que les génotypes de piment tolérants au sel sont capables de maintenir un rapport $\mathrm{K}^{+} / \mathrm{Na}^{+}$élevé. Cette sélectivité importante est réalisée par la réduction de l'absorption de $\mathrm{Na}^{+}$et la promotion de l'absorption de $\mathrm{K}^{+}$(Haouala et al., 2007). Le maintien d'un rapport

\section{MATERIEL ET METHODES,}

Matériel végétal: Trois variétés locales de piment appartenant à la forme cultivée Capsicum annuum L. sont utilisées: Deux variétés piquantes Beldi (longueur moyenne des fruits $15 \mathrm{~cm}$, poids moyen $=33 \mathrm{gr}$ ) et Midass (longueur moyenne $=5 \mathrm{~cm}$ et poids moyen du fruit $=5 \mathrm{~g}$ ) cultivées respectivement en culture de saison en plein champ en été au sahel (Centre -Est de la Tunisie) et dans les oasis d'El jerid au sud Tunisien (Sud-est de la Tunisie). Un poivron de type long « Nabeul » (longueur moyenne du fruit $=25 \mathrm{~cm}$ et poids moyen du fruit $=60 \mathrm{gr}$ ) cultivé essentiellement dans la région du cap Bon (Nordest de la Tunisie) sous abri-serres plastiques.

Conduite de la culture : Les expérimentations ont été réalisées à l'Institut National de la Recherche Agronomique de Tunisie durant l'année 2008-2009. Le semis des trois variétés est effectué au début du mois de septembre en terrines remplies de tourbe et placées dans une chambre à $25^{\circ} \mathrm{C}$. II est suivi, à environ 25 jours, d'un repiquage en plaques alvéolaires. La culture dure 30 jours. Elle est conduite à une température de $18-20^{\circ} \mathrm{Cla}$ nuit et $28^{\circ} \mathrm{C}$ le jour, et sous une humidité relative de $70 \%$. Au stade 4-5 feuilles, les plantes sont transférées dans des pots en plastique de $30 \mathrm{~cm}$ de diamètre et de $50 \mathrm{~cm}$ de profondeur contenant chacun un substrat composé de terre de jardin, de fumier de ferme décomposé et de sable grossier à proportions égales. Les conteneurs de culture sont placés sous abri-serre plastique. Dans la région de Tunis (Tunisie), les températures moyennes enregistrées sous l'abri-serre durant la période de la culture (novembre 2008- avril 2009) sont situées entre 16,3 et $27,6^{\circ} \mathrm{C}$. Au début, l'arrosage des graines et des jeunes plantules est effectué à l'eau courante et exécuté sous formes de fines gouttelettes pour éviter le de sélectivité élevé sur des plantes de poivron est due à la présence des ions $\mathrm{Ca}^{2+}$ (Rubio et al , 2003). La capacité des génotypes à expulser $\mathrm{I} \mathrm{Na}^{+}$à partir des racines dans le milieu de croissance et à maintenir un ratio $\mathrm{K}^{+} / \mathrm{Na}^{+}$élevé est une expression de la tolérance au sel (Yoshida, 2002; Zhu, 2002). Pour Munns et James (2003), le mécanisme d'exclusion de $\mathrm{Na}^{+}$est étroitement corrélé avec la tolérance à la salinité chez des génotypes de blé tétraploïde. L'objectif du présent travail est d'étudier l'effet de la salinité sur la croissance, la conductance stomatique, la teneur en chlorophylle et sur la nutrition minérale chez trois variétés de piment.

déchaussement des graines et la casse de jeunes tiges. Cette eau a une conductivité électrique de $\mathrm{CE}=$ $0,73 \mathrm{mmhos} / \mathrm{cm}$. Une fois les plantules acclimatées $(7$ jours après la plantation), l'application des traitements salins a commencé et l'irrigation est effectuée à l'eau courante additionnée ou non de différentes doses de $\mathrm{NaCl}$ (T1: $0 \mathrm{mM} ; \mathrm{T} 2: 25 \mathrm{mM}$, T3: $50 \mathrm{mM}$ et T4: $100 \mathrm{mM}$ de $\mathrm{NaCl}$ ). Le milieu témoin est arrosé uniquement à l'eau courante. Les arrosages sont effectués de manière à maintenir le substrat de la culture à la capacité au champ. Les irrigations avec les solutions salines ont commencé le 7 novembre 2008 et ont été terminées le 30 avril 2009. Dispositif expérimental : Le dispositif expérimental est de type factoriel à deux facteurs en randomisation totale. Le premier facteur représente les quatre traitements salins (T1: $0 \mathrm{mmol}, \mathrm{T} 2: 25 \mathrm{mmol}, \mathrm{T} 3: 50 \mathrm{mmol}$ et T4: 100 mmolde $\mathrm{NaCl}$ ) et le 2 ème facteur est représenté par les trois variétés (Beldi, Midass et Nabeul). Le nombre de répétition est de cinq. Chaque répétition comporte trois pots par variété et par traitement.

Paramètres mesurés: -La matière sèche (MS) de la plante (partie aérienne et racinaire) est déterminée à la fin de l'expérimentation par pesée à l'aide d'une balance de précision de type Mettler P3 après séchage des plantes à l'étuve à $80^{\circ} \mathrm{C}$ pendant $72 \mathrm{~h}$.

- La conductance stomatique est mesurée (Mi mars) par un poromètre (Decagon dvices, Pullman, WA) durant un jour clair entre $10 \mathrm{~h}$ et 12h. La valeur moyenne calculée par variété et par traitement correspond à trois mesures effectuées sur des feuilles appartenant aux deux derniers étages foliaires. 
- - La teneur en chlorophylle est déterminée selon la méthode de Strain and Svec, (1966) sur trois plantes par variété et par traitement. Des échantillons de $1 \mathrm{gr}$ de MF chacun sont prélevés sur la plus jeune feuille complètement développée. L'extraction de la chlorophylle a été faite avec de l'acétone à $90 \%$ et la lecture à l'aide d'un spectrophotomètre UV / visible à 663 et $645 \mathrm{~nm}$.

Les concentrations en chlorophylle sont calculées selon les formules suivantes (Strain and Svec, 1966):

Chl a $\left(\mathrm{mg}^{\mathrm{m}} \mathrm{ml}^{-1}\right)=11,64 \times\left(\mathrm{A}_{663}\right)-2,16 \times\left(\mathrm{A}_{645}\right)$

Chl b $\left(\mathrm{mg}^{\mathrm{m}} \mathrm{ml}^{-1}\right)=20,97 \times\left(\mathrm{A}_{645}\right)-3,94 \times\left(\mathrm{A}_{663}\right)$

Chl total $\left(\mathrm{mg} \cdot \mathrm{ml}^{-1}\right)=\mathrm{chl} \mathrm{a}+\mathrm{chl} \mathrm{b}$

\section{RESULTATS}

Matière sèche : La production de matière sèche des feuilles et des racines de piment a diminué significativement avec l'augmentation de la salinité de l'eau d'irrigation (figure 1). Les variétés de piment ont répondu différemment à la concentration en sel. En effet, la variété Nabeul est la plus touchée par le sel. Les diminutions de la biomasse des feuilles et des racines observées sont respectivement de 28 et $43 \%$ à $25 \mathrm{mmol}$;
$A_{663}$ et $A_{645}$ représentent la valeur d'absorbance mesurée par spectrophotométrie aux longueurs d'ondes.

-Les teneurs en calcium, sodium et potassium sont déterminées par un spectrophotomètre à flamme (Eppendorf) à la fin de l'expérimentation. Les plantes sont divisées en feuilles et racines et séchées pendant $72 \mathrm{~h}$ dans un four à air chaud à $80^{\circ} \mathrm{C}$. Après broyage, les échantillons ont été incinérés à $550^{\circ} \mathrm{C}$ pendant $4 \mathrm{~h}$ et dissous dans du $\mathrm{HCl}$ à $1 \%$ et analysés pour les ions $\mathrm{Na}^{+}, \mathrm{Ca}^{2+}$ et $\mathrm{K}{ }^{+}$.

Étude statistique : L'analyse de la variance ANOVA a été faite par le programme SAS (2000). Chaque donnée constitue la moyenne d'au moins trois mesures. Pour la comparaison des moyennes, le test de Newman Keuls au seuil de $5 \%$ a été utilisé.

de 61,4 et $72 \%$ à $50 \mathrm{mmol}$ et de 70 et $86 \%$ à $100 \mathrm{mmol}$ La variété Midass est la moins affectée par le sel puisque les réductions de matière sèche ne commencent à se faire sentir qu'à partir de $50 \mathrm{mmol}$ pour les feuilles $(28 \%$ de réduction) et $100 \mathrm{mmol}$ pour les racines $(70 \%$ de réduction). Il est à remarquer même qu'une augmentation de la biomasse des feuilles a été constatée pour cette variété pour la concentration de $25 \mathrm{mmol}$ de $\mathrm{NaCl}$.
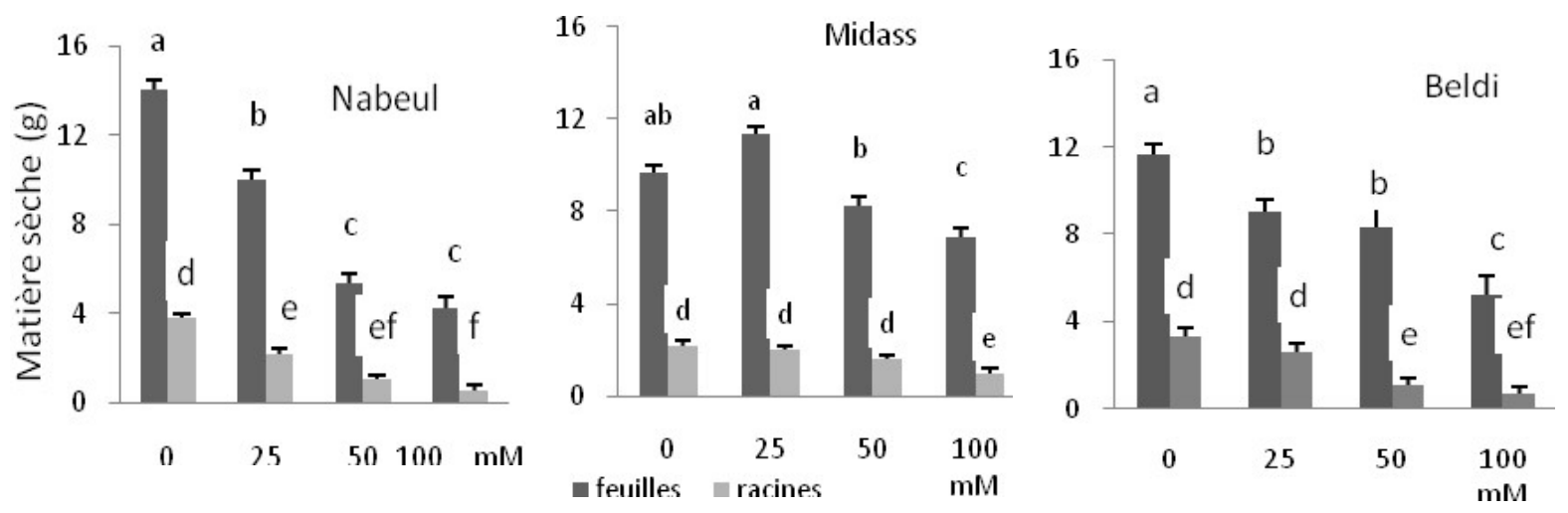

Figure 1. Effet du stress salin sur les productions de matières sèches des feuilles et des racines chez trois variétés de piment. Effect of salt stress on the production of leaves and roots dry matter of the different studied pepper varieties

Conductance stomatique : La conductance stomatique a été significativement réduite sous l'effet du stress salin. En effet, tous les génotypes ont été affectés avec cependant une chute plus prononcée chez les variétés Beldi et Nabeul respectivement de 17et 25\% pour
T2 : 42 et $52 \%$ pour T3: et 57 et $66,74 \%$ pour T4: (figure 2). La variété Midass semble la moins affectée par la salinité puisque la réduction est seulement de $30 \%$ pour le traitement le plus sévère (figure 2). 

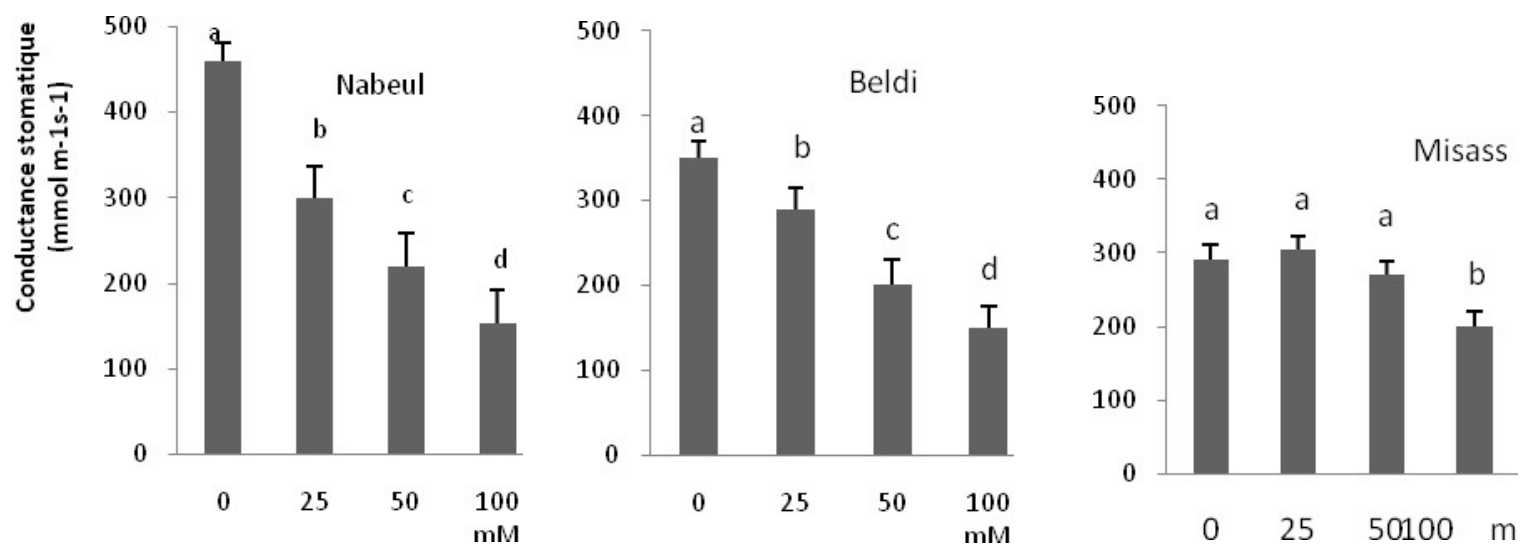

Figure2. Effet du stress salin sur la conductance stomatique chez trois variétés de piment. Effect of salt stress on leaves stomatal conductance of the different studied pepper varieties

Teneur en chlorophylle : Les teneurs en chlorophylle a, chlorophylle $b$ et en chlorophylle totale ont été significativement réduites sous l'effet du stress salin (Tableau 1). Toutes les variétés ont été affectées avec cependant une chute plus prononcée chez la variété Nabeul pour la chlorophylle a (32\% de réduction en $\mathrm{T} 3$ et $67 \%$ en T4) et la chlorophylle totale (36 \% de réduction en T3 et $65 \%$ en T4). La concentration de la chlorophylle a a été trois fois plus élevée que la concentration de la chlorophylle b. Midass a été la variété la moins affectée par la salinité ( $24 \%$ de réduction pour T4). Au contraire, on observe même une augmentation des chlorophylles a et totale pour le traitement $\mathrm{T} 3$ de $\mathrm{NaCl}$.

Tableau.1 : Teneurs des trois variétés de piment en chlorophylle $a$, chlorophylle $b$ et chlorophylle totale à différents niveaux de salinité. Contents of chlorophyll ( $a, b$ and total) in leaves of the studied pepper varieties under different salinity treatments.

\begin{tabular}{|c|c|c|c|c|}
\hline Variétés & traitement salin & $\begin{array}{l}\text { Chla } \\
\text { (mg }\end{array}$ & $\begin{array}{l}\text { Chlb } \\
\text { MF) }\end{array}$ & Chl total \\
\hline Beldi & $\begin{array}{c}0 \\
25 \\
50 \\
100\end{array}$ & $\begin{array}{c}2,80 \mathrm{a} \\
2,64 \mathrm{a} \\
2,87 \mathrm{a} \\
2,01 \mathrm{~b}\end{array}$ & $\begin{array}{c}0,85 a \\
0,78 a \\
1,02 a \\
0,53 b\end{array}$ & $\begin{array}{l}3.65 \mathrm{a} \\
3,42 \mathrm{a} \\
3,89 \mathrm{a} \\
2,54 \mathrm{~b}\end{array}$ \\
\hline Nabeul & $\begin{array}{c}0 \\
25 \\
50 \\
100\end{array}$ & $\begin{array}{l}5,05 \mathrm{a} \\
4,66 \mathrm{~b} \\
3,42 \mathrm{c} \\
1,64 \mathrm{~d}\end{array}$ & $\begin{array}{l}1,85 a \\
1,12 b \\
0,98 b \\
0,71 b\end{array}$ & $\begin{array}{l}6,90 \mathrm{a} \\
5,78 \mathrm{~b} \\
4,40 \mathrm{c} \\
2,35 \mathrm{~d}\end{array}$ \\
\hline Midass & $\begin{array}{r}0 \\
25 \\
50 \\
100\end{array}$ & $\begin{array}{l}3,62 \mathrm{a} \\
3,23 \mathrm{~b} \\
4,17 \mathrm{a} \\
2,72 \mathrm{c}\end{array}$ & $\begin{array}{l}0,98 \mathrm{a} \\
0,85 \mathrm{a} \\
1,03 \mathrm{a} \\
0,48 \mathrm{a}\end{array}$ & $\begin{array}{l}4,6 \mathrm{ab} \\
4,04 \mathrm{~b} \\
5,20 \mathrm{a} \\
3,20 \mathrm{c}\end{array}$ \\
\hline
\end{tabular}

Les valeurs de le même colonne affectées de lettres différentes sont significtaivement différentes au seuil de $5 \%$. Values in the same column marked with different letter are significantly different at $5 \%$

T

eneur en éléments minéraux : L'effet du stress salin sur la teneur en $\mathrm{Ca}^{2+}$ et en $\mathrm{K}^{+}$a varié avec les variétés et la charge en sel de l'eau d'irrigation. Nous avons remarqué deux sortes de comportements qui peuvent être résumés comme suit (tableau 2) :
- L'analyse statistique n'a pas montré de différence significative pour les teneurs en $\mathrm{Ca}^{2+}$ et en $\mathrm{K}^{+}$chez la variété Midass quelque soit le traitement salin appliqué.

- Les taux potassique et calcique des feuilles ont diminué sous l'effet de la salure essentiellement 
pour la variété Nabeul où la réduction de $\mathrm{Ca}^{2+}$ est respectivement de $33 \%$ pour T2 et de $65 \%$ pour T3. Pour l'ion $\mathrm{K}^{+}$, cette chute est de $42 \%$ pour T3 (Tableau 2).

- - Pour la variété Beldi, l'analyse statistique n'a pas montré de différence significative pour la teneur en $\mathrm{Ca}^{2+}$ mais une réduction significative en $\mathrm{K}^{+}$à partir de $50 \mathrm{mmol}$.

De plus, le stress salin a provoqué une augmentation significative de la teneur en $\mathrm{Na}^{+}$chez toutes les variétés. Cette augmentation est proportionnelle à la concentration en sel.

Les valeurs les plus faibles ont été enregistrées chez la variété Midass, où on note un effet significatif seulement pour le traitement le plus stressé T4 (10,2 mg/g-1 MS) (tableau2).

Pour Beldi, la teneur en $\mathrm{Na}^{+}$commence à augmenter significativement dans les feuilles à partir de $50 \mathrm{mM}$ de $\mathrm{NaCl}$. En effet, les teneurs en $\mathrm{Na}^{+}$sont 5 et 8 fois plus élevées à 50 et $100 \mathrm{mM}$ par rapport au témoin $(3,8 \mathrm{mg} . \mathrm{g}$ 1). Pour $\mathrm{Nabeul}$, l'ion $\mathrm{Na}^{+}$s'accumule rapidement dans les feuilles sous l'effet de la salinité à partir de $25 \mathrm{mmol}$ de $\mathrm{NaCl}$

(15 mg.g-1 MS contre 4 mg.g-1 chez le témoin). Puis, la teneur de ce cation devient 7 et 10 fois plus élevée quand la concentration du milieu passe de 50 à $100 \mathrm{mmol}$ de $\mathrm{NaCl}$ (tableau2).

Tableau 2. Teneurs des trois variétés de piment en calcium $\left(\mathrm{Ca}^{2+}\right)$, potassium $\left(\mathrm{K}^{+}\right)$et en sodium $\left(\mathrm{Na}^{+}\right)$total à différents niveaux de salinité. Leaf mineral analysis of three varieties of pepper irrigated with solutions at four salinity levels. Mineral contents $\left(\mathrm{Ca}{ }^{2+}, \mathrm{K}^{+}\right.$and $\left.\mathrm{Na}^{+}\right)$in leaves of the studied pepper varieties under different salinity treatments

\begin{tabular}{lccll}
\hline Variétés & traitement salin $(\mathrm{mM})$ & $\mathrm{Ca}^{2+}\left(\mathrm{mg} \mathrm{g}^{-1}\right)$ & $\mathrm{K}^{+}\left(\mathrm{mg} \mathrm{g}^{-1}\right)$ & $\mathrm{Na}+\left(\mathrm{mg} \mathrm{g}^{-1}\right)$ \\
\hline Beldi & 0 & $36,90 \mathrm{a}$ & $48,60 \mathrm{a}$ & $3,80 \mathrm{c}$ \\
& 25 & $38,00 \mathrm{a}$ & $46,10 \mathrm{a}$ & $4,60 \mathrm{c}$ \\
& 50 & $35,50 \mathrm{a}$ & $38,50 \mathrm{~b}$ & $21,00 \mathrm{~b}$ \\
& 100 & $33,00 \mathrm{a}$ & $35,30 \mathrm{~b}$ & $31,20 \mathrm{a}$ \\
Nabeul & & & \\
& 0 & $37,80 \mathrm{a}$ & $48,60 \mathrm{a}$ & $4,00 \mathrm{~d}$ \\
& 25 & $25,20 \mathrm{~b}$ & $46,10 \mathrm{a}$ & $15,00 \mathrm{c}$ \\
& 50 & $13,10 \mathrm{C}$ & $28,50 \mathrm{~b}$ & $29,00 \mathrm{~b}$ \\
Midass & 100 & $10,20 \mathrm{c}$ & $25,30 \mathrm{~b}$ & $39,70 \mathrm{a}$ \\
& & & & \\
& 0 & $41,30 \mathrm{a}$ & $48,70 \mathrm{a}$ & $2,50 \mathrm{~b}$ \\
& 25 & $42,80 \mathrm{a}$ & $46,10 \mathrm{a}$ & $4,20 \mathrm{~b}$ \\
& 50 & $38,50 \mathrm{a}$ & $45,30 \mathrm{a}$ & $4,50 \mathrm{~b}$ \\
& 100 & $36,60 \mathrm{a}$ & $45,10 \mathrm{a}$ & $10,20 \mathrm{a}$ \\
\hline
\end{tabular}

Les valeurs de le même colonne affectées de lettres différentes sont significtaivement différentes au seuil de $5 \%$. Values in the same column marked with different letter are significantly different at $5 \%$

\section{DISCUSSION}

Croissance végétative: La croissance végétative (Matière sèche feuilles+ racines) est sévèrement affectée par la salinité à partir de $50 \mathrm{mM} \mathrm{NaCl}$. Cette diminution a touché les trois génotypes de piment essentiellement la variété Nabeul, où la réduction de la biomasse commence même à $30 \mathrm{mM}$ de $\mathrm{NaCl}$. Ceci corrobore avec les résultats de Lycoskoufis et al.(2005), Navarro et al.,(2010) et Niu et al.,(2010 ) sur le piment et confirme les investigations qui indiquent que le piment est une espèce sensible au sel (Sonneveld, 1988; Navarroet al., 2002). Cependant, cette sensibilité au sel a touché plus la partie racinaire que la partie aérienne. ceci est en accord avec les observations de Hamrouni et al.(2011) qui a indiqué que la plante s'adapte au stress salin en réduisant en premier lieu son système racinaire préservant ainsi la partie aérienne afin de maintenir et d'assurer la production des photosynthétats. Cette diminution observée chez les plantes de piment est expliquée par le fait que $\mathrm{NaCl}$ agit en augmentant la pression osmotique du milieu, ce qui empêche l'absorption en eau par le système racinaire (Marshner, 1995, Navarro et al. 2002) et entraine par conséquent, une réduction de la croissance. Sur le plan cellulaire, la diminution de la fabrication de la matière sèche est due à une baisse du nombre de divisions cellulaires (Benamar, 2009).

Conductance stomatique : La conductance stomatique a été significativement réduite sous l'effet du stress salin surtout chez la variété Nabeul suivie de Beldi. Ces résultats rejoignent ceux de Lycoskoufis et al.(2005) et 
Niu et al.,(2010) sur le piment et Baker and Rosenqvist ( 2004) sur la tomate qui ont signalé que la salinité peut limiter la photosynthèse nette et la conductance stomatique soit en raison d'une limitation de l'offre de $\mathrm{CO}_{2}$ résultant de la fermeture partielle des stomates (fonction stomatique) soit en altérant le mécanisme biochimique de la fixation du $\mathrm{CO}_{2}$ (fonction non stomatique), ou par les deux procédures.

Teneur en Chlorophylle : La teneur en chlorophylle est significativement réduite par la salinité surtout pour le traitement sévère $T 4$. Ces résultats corroborent avec les observations de Cengiz et al (2009) sur le piment. . Selon Feigin et al. (1991); Grattan and Grieve, (1994), NaCl a un effet antagoniste sur l'absorption de l'azote (N) qui est une composante essentielle de la structure de la molécule de chlorophylle. Chez la variété Midass, on a observé une augmentation des chlorophylles a et totale pour le traitement T3:50 mM de $\mathrm{NaCl}$. Ces résultats rejoignent ceux de Sharaf et al(1990) sur la tomate et Dali et al. (1997) sur le piment qui ont indiqué qu'une salinité modérée augmente la quantité de la chlorophylle a et de la chlorophylle totale.

Nutrition minérale : La tolérance au sel chez les plantes supérieures dépend de la façon dont les plantes contrôlent le transport du sel à travers les organes (Niu et al, 2010). En effet, les mécanismes de tolérance au sel sont de trois types distincts chez les plantes : celles qui tolèrent le stress osmotique, celles qui excluent le $\mathrm{Na}^{+}$et le $\mathrm{Cl}^{-}$de leurs tissus et celles qui tolère l'accumulation de $\mathrm{Na}^{+}$et $\mathrm{Cl}$ - dans leurs tissus (Haouala 2007, Munns and tester, 2008). Cette sensibilité ou cette tolérance à la salinité varie selon les espèces et les variétés. Dans cette étude, la sensibilité élevée de la variété Nabeul à la salinité indique que celle ci ne tolère pas une accumulation élevée de $\mathrm{Na}^{+}$dans ses tissus. En effet, c'est la variété la plus touchée par le sel. La variété la plus tolérante est Midass, elle a la plus faible teneur en $\mathrm{Na}^{+}$, ce qui indique qu'elle est de type « excluder ». Elle développe des mécanismes pour limiter l'accumulation de $\mathrm{Na}^{+}$dans ses tissus (Maeshner, 1995). Beldi tient une place intermédiaire entre Midass et Nabeul, ceci indique que ce cultivar a une certaine tolérance au sodium mais pas aussi importante que Midass.

La Salinité peut affecter l'absorption de $\mathrm{Ca}^{2+}$ et $\mathrm{K}^{+}$, en fonction des espèces et du niveau de salinité (Grattan et
Grieve, 1999). Dans cette étude, les concentrations de $\mathrm{Ca}^{+}$et de $\mathrm{K}^{+}$dans les feuilles varient selon les variétés. Pour la plus sensibles au sel « Nabeul », elle a à la fois des teneurs en $\mathrm{Ca}^{2+}$ et $\mathrm{K}^{+}$, plus faibles lorsque les plantes ont été irriguées avec une eau chargée par rapport au témoin. Pour Beldi, la seconde variété sensible au sel, la concentration de $\mathrm{K}+$ des feuilles a été réduite par la salinité élevée, mais sa concentration en $\mathrm{Ca}^{2+} n$ 'a pas été affectée. Pour Midass, la plus tolérante à la salinité, la teneur en $\mathrm{Ca}^{2+}$ et $\mathrm{K}^{+}$des n'a pas été affectée par les traitements salins. Al-Karaki et al. (2009) et Hamrouni et al. (2011), en quantifiant respectivement la réponse de trois cultivars de poivrons aux différents niveaux de alinité, ont constaté que les génotypes tolérants seraient capables d'accumuler de grandes quantités de potassium particulièrement dans les parties aériennes par rapport aux cultivars sensibles. L'efficacité d'absorption et d'utilisation du $\mathrm{K}^{+}$, qui agit comme osmoticum, est donc capitale dans l'adaptation au stress salin (Hamrouni et al, 2011 ; Niu et al. 2010 ; Aktas et al.2006) ont rapporté que les génotypes de piment tolérants au sel accumulent moins de $\mathrm{Na}^{+}$dans les feuilles que les génotypes sensibles. Ces études indiquent que les génotypes ayant la capacité d'exclure $\mathrm{Na}^{+}$sont généralement plus tolérants à la salinité.

Evolution du rapport de sélectivité $\mathrm{K}^{+} / \mathrm{Na}^{+}$: Le rapport de sélectivité $\mathrm{k}^{+} / \mathrm{Na}^{+}$a diminué dans les feuilles des trois variétés (figure 3 ) suite à l'augmentation de la salinité. Cramer et al. (1985) ont démontré qu'en présence de concentrations élevées de $\mathrm{NaCl}, \mathrm{Na}+$ déplace $\mathrm{Ca}^{2+} \mathrm{du}$ plasmalemme des cellules racinaires, ce qui entraine l'augmentation de la perméabilité de la membrane et provoque un efflux du $\mathrm{K}^{+}$et une altération du rapport de sélectivité $\mathrm{K}^{+} / \mathrm{Na}^{+}$. Au niveau des feuilles, le caractère $\mathrm{K}^{+} / \mathrm{Na}^{+}$a été plus discriminant entre les variétés. Notons que , même avec l'accumulation du $\mathrm{Na}^{+}$, la variété Midass a présenté le rapport de sélectivité le plus élevé par rapport à la variété Beldi suivie de Nabeul où on observe un rapport de sélectivité très faible à une salinité modéré $(25 \mathrm{mM})$. Ceci suggère que les plantes qui réussissent leur croissance en milieu salin sont celles qui maintiennent un rapport $\mathrm{K}^{+} \mathrm{Na}^{+}$plus élevé dans leur cytoplasme que dans la rhizosphère (El-lklil et al., 2001). 


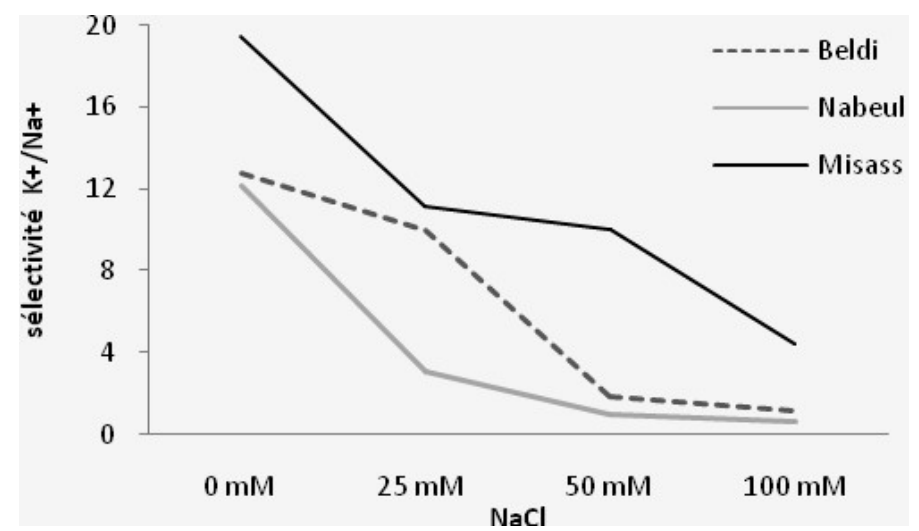

Figure 3 : Evolution du rapport de sélectivité $(\mathrm{K}+/ \mathrm{Na}+)$ dans les feuilles de tois variétés de piment sous différents régimes de salinité. Evolution of the selectivity ratio in the leaves of the three pepper varieties under different salinity treatments

\section{CONCLUSION}

Les résultats de cette expérimentation ont permis de rendre compte des différences génotypiques vis-à-vis de la salinité. En effet, la variété Nabeul qui est de type piment doux est plus sensible au sel que les variétés Beldi et Midass. Cette dernière qui est très piquante s'est montrée plus tolérante à la salinité vu qu'elle n'a été

\section{BIBLIOGRAPHIE}

Abdel Latef, A.A., 2010. Changes of antioxidative enzymes in salinity tolerance among different wheat cultivars. Cereal Res. Comm. 38, 43-55.

Aktas, H., Abak, K., Cakmak, I., 2006. Genotypic variation in the response of pepper to salinity. Sci. Hortic. 110, 260-266.

Al-Karaki, G., Al-Ajmi, A., Othman, Y., 2009. Response of soilless grown sweet pepper cultivars to salinity. Acta Hortic. 807, 227-231.

Baker, N.R., Rosenqvist, E., 2004. Applications of chlorophyll fluorescence can improve crop production strategies: an examination of future possibilities. J. Exp. Bot. 55, 1607-1621

Benamar B., Daguin F. \& Kaid-Harche M., 2009.Effet du stress salin sur la germination et la croissance in vitro du pistachier (Pistacia vera L.). Comptes Rendus Biologies, 332, 752-758.

Cabanero, F.J., Martınez, V., Carvajal, M., 2004. Does calcium determine water uptake under saline conditions in pepper plants, or is it water flux, which determines calcium uptake? Plant Sci. 166, 443-450.Comptes Rendus Biologies, 332, 752-758.

Cengiz Kaya, Muhammed Ashraf, Osman Sonmez, Salih Aydemir, Atilla Levent Tuna, Mehmet Ali Cullu,.2009. The influence of arbuscular affectée que lorsque la dose de sel est élevé (supérieur $50 \mathrm{mmol}$ ). Ce comportement adaptatif est dû à l'origine de l'écotype Midass qui est cultivé essentiellement au sud tunisien et où la charge en sel de l'eau d'irrigation dépasse $3,5 \mathrm{~g} / \mathrm{l}(60 \mathrm{mM})$ (Slama, 2004) et à la présence probablement de la capsaicine.

mycorrhizal colonisation on key growth parameters and fruit yield of pepper plants grown at high salinity. Scientia Horticulturae 121, 1-6.

Chartzoulakis, K., Klapaki, G., 2000. Response of two greenhouse pepper hybrids to $\mathrm{NaCl}$ salinity during different growth stages. Sci. Hortic. 86, 247-260.

Cornillon P. \& Palloix A., 1997, Influence of sodium chloride on the growth and mineral nutrition of pepper cultivars. Journal of Plant Nutrition, 20, 1085-1094.

Cramer, G.R., Lauchli, A., Polito, V.S., 1985. Displacement of $\mathrm{Ca} 2+$ by $\mathrm{Na}+$ from the plasmalema of root cells. A primary response to salt stress. Plant Physiol 79: 207-211.

Dali, N., Ben Ghanem, H., Mougou, A., Ben Taeib, T., 1997. Effet d'un stress salin sur la répartition entre amidon et sucres solubles dans les feuilles de deux lignées de tomate. Revue de l'INAT, Journal des Sciences Agronomique 12 (1), 131147.

El-Iklil, Y., Karrou, M., Mrabet, R. et Benichou, M. 2002. Effet du stress salin sur la variation de certains metabolites chez Lycopersicon esculentum et 
Lycopersicon sheesmanii. Can. J. Plant Sci. 82: 177-183.

Feigin, A., Pressman, E., Imas, P., Milta, O., 1991. Combined effects of KNO3 and salinity on yield and chemical composition of lettuce and Chinese cabbage. Irrig.Sci. 12, 223-230.

Ghassemi, F., Jakeman, A.J., Nix, H.A., 1995. Salinisation of land and water resources: human causes, extent, management and case studies. Wallingford, Oxon, UK.

Grattan, S.R., Grieve, C.M., 1994. Mineral nutrient acquisition and response by plants grown in saline environments. In: Pessarakli, M. (Ed.), Handbook of Plant and Crop Stress. Marcel Dekker, New York, pp. 203-226.

Hamrouni L., Hanana M., Abdelly C. et Ghorbel A., 2011. Exclusion du chlorure et inclusion du sodium : deux mécanismes concomitants de tolérance à la salinité chez la vigne sauvage vitis vinifera subsp.sylvestris (var.'sejnène') Bitechnol. agronom. Soc. Environ,15 (3), 387-400.

Haouala, F., Ferjani, H., Ben El Hadj, S., 2007. Effet de la salinité sur la répartition des cations $(\mathrm{Na}+, \mathrm{K}+$ et $\mathrm{Ca} 2+$ ) et du chlore ( $\mathrm{Cl}-$ ) dans les parties aériennes et les racines du ray-grass anglais et du chiendent. Bitechnol. agronom. Soc. Environ, 11 (3), 235-244.

Lycoskoufis, I.H., Savvas, D.,Mavrogianopoulos,.G,2005. Growth, gas exchange, and nutrient status in pepper (Capsicum annuum L.) grown in recirculating nutrient solution as affected by salinity imposed to half of the root system. Scientia Horticulturae 106, 147-161.

Mansour, M.M.F., 2003. Transport proteins and salt tolerance in plants. Plant Sci. 164, 891-900.

Marschner, H., 1995. Saline soils, Mineral Nutrition of Higher Plants, 2nd ed. Academic Press, San Diego, CA.

Munns, R., James, R.A., 2003. Screening methods for salinity tolerance: a case study with tetraploid wheat. Plant Soil 253, 201-218.

Munns, R., Tester, M., 2008. Mechanisms of salinity tolerance. Annu. Rev. Plant Biol. 59, 651-681.

Navarro, J.M., Garrido, C., Carvajal, M., Martinez, V., 2002. Yield and fruit quality of pepper plants under sulphate and chloride salinity. J. Hort. Sci. Biotech. 77, 52-57.

Navarro, J.M., Garrido, C., Martinez, V., Carvajal, M., 2003. Water relations and xylem transport of nutrients in pepper plants grown under two different salts stress regimes. Plant Growth Regul. 41, 237-245.

Navarro J.M., Garrido C., flores p. \& Martinez V., 2010, The effect of salinity on yield and fruit quality of pepper grown in perlite. Spanish Journal of Agricultural Research, 8, 142-150.

Niu, G., Rodriguez, D.S., Starman, T., 2010. Response of bedding plants to saline water irrigation. Hort Science 45 (4), 628-636.

Pasternak, D., Malach, Y.D., 1994. Crop irrigation with saline water. In: Pessarakli Mo (Ed.), Handbook of Plant and Crop Stress. Marcel Dekker, New York, pp.599-622.

Rengel, Z., 1992. The role of calcium in salt toxicity. Plant Cell Environ. 15, 625-632.

Rozema, J., Flowers, T., 2008. Crops for a salinized world. Science 322, 1478-1480.

Rubio, F., Flores, P., Navarro, J.M., Martinez, V., 2003. Effects of $\mathrm{Ca}^{2+} \mathrm{K}^{+}$and cGMP on $\mathrm{Na}^{+}$uptake in pepper plants. Plant Sci. 165, 1043-1049.

Rubio, J.S., Rubio, F., Martínez, V., García-Sánchez. F, 2010. Amelioration of salt stress by irrigation management in pepper plants grown in coconut coir dust. Agricultural Water Management 97, 1695-1702.

Shannon, M.C., Grieve, C.M., 1999. Tolerance of vegetable crops to salinity. Sci. Hortic. 78, 5-38.

Sharaf, A., Labib, S. et El Massry, R., 1990. Effect of kinetin on the biochemical constituents of tomato plants under different levels of salilnity. Zagazig Journal of Agricultural Research (Egypt).12: 417-441.

Sheng, M., Tang, M., Chan, H., Yang, B., Zhang, F., Huang, Y., 2008. Influence of arbuscular mycorrhizae on photosynthesis and water status of maize plants under salt stress. Mycorrhiza 18, 287-296.

Slama F., 2004. La salinité et la production végétale. Centre de publication universitaire, Tunis.163P.

Sonneveld, C., 1988. The salt tolerance of greenhouse crops. Neth. J. Agric. Sci. 36, 63-73.

Strain, H.H., Svec, W.A, 1966. Extraction, separation, estimation and isolation of chlorophylls. IN: Vernon, L.P, Seeley, G. R (EDS. The chlorophylls. Academic press, pp, 21-66.

Villa-Castorena, M., Ulery, A.L., Catalan-Valencia, E.A., Remmenga, M.D., 2003. Salinity and nitrogen rate effects on the growth and yield of Chile pepper plants. Soil.Sci. Soc. Am. J. 67, 17811789. 
Yoshida, K., 2002. Plant biotechnology genetic engineering to enhance plant salt tolerance. J. Biosci. Bioeng. 94, 585-590.

Zeng, L., Poss, J., Wilson, C., Draz, A.S.E., Grieve, C.M., 2003. Evaluation of salt tolerance in rice genotypes by physiological characters. Euphytica 129, 281-292.

Zid E. \& Grignon C., 1991, Les tests de sélection précoce pour la résistance des plantes aux stress. Cas des stress salin et hydrique. L'amélioration des plantes pour l'adaptation aux milieux arides,
AUPELFUREF. Jon Libbey Eurotext, Paris, 91108.

Zhu, J.K., 2002. Salt and drought stress signal transduction in plants. Ann. Rev. Plant Biol. 53, 247-273.

Zribi, L., Gharbi, F., Rezgui, F., Rejeb, S., Nahdi, H., Rejeb, M.N., 2009. Application of chlorophyll fluorescence for the diagnosis of salt stress in tomato "Solanum lycopersicum (variety Rio Grande)". Scientia Horticulturae 120, 367-372. 